\title{
THE INDEX OF HARMONIC FOLIATIONS ON SPHERES ${ }^{1}$
}

\author{
BY
}

FRANZ W. KAMBER AND PHILIPPE TONDEUR

\begin{abstract}
For foliations on a compact oriented manifold there is a natural energy functional, defined with respect to a Riemannian metric. Harmonic Riemannian foliations are then the critical foliations for this functional under an appropriate class of special variations. The index of the title is the index of the Hessian of the energy functional at a critical, i.e., harmonic foliation. It is a finite number. In this note it is shown that for a harmonic Riemannian foliation $\mathscr{F}$ of codimension $q$ on the $n$-sphere $(n>2)$ this index is greater or equal to $q+1$. Thus $\mathscr{F}$ is unstable. Moreover the given bound is best possible.
\end{abstract}

Introduction. For foliations on a compact oriented manifold $M$ there is a natural energy functional, defined with respect to a Riemannian metric $g_{M}$ on $M[4,5]$. Harmonic Riemannian foliations are then the critical foliations for this functional under an appropriate class of so-called special variations, defined by sections of the normal bundle $Q[4,5]$. For foliations defined by the inverse images of submersions, harmonic foliations correspond to harmonic maps in the sense of Eells and Sampson [2].

The Hessian of the energy functional can be evaluated by the following fundamental formula, which is proved in the appendix to [7].

SECOND VARIATION FORMULA. Let $M$ be a compact oriented manifold, and $\mathcal{F}$ a Riemannian and harmonic foliation with respect to a bundle-like metric $g_{M}$. Consider the 2-parameter family $\mathcal{F}_{s, t}$ of special variations of $\mathscr{F}=\mathscr{F}_{0,0}$ defined by two sections $\mu, \nu$ of the normal bundle $Q$. Then for the second derivative of the energy we have

$$
\left.\frac{\partial^{2} E\left(\mathscr{F}_{s, t}\right)}{\partial s \partial t}\right|_{s=0, t=0}=\left\langle\left(\Delta-\rho_{\nabla}\right) \mu, \nu\right\rangle .
$$

The sections $\mu, \nu \in \Gamma Q$ define a 2-parameter special variation $\mathscr{F}_{s, t}$ of $\mathscr{F}_{0,0}=\mathscr{F}$ through Riemannian foliations by patching the local data

$$
\Phi_{s, t}^{\alpha}(x)=\exp _{t^{\alpha}(x)}\left(s \mu^{\alpha}(x)+t \nu^{\alpha}(x)\right) .
$$

Here $f^{\alpha}$ is a local submersion defining $\mathscr{F}$ in an open set $U_{\alpha} . \Phi_{s, t}^{\alpha}$ is then the local submersion defining $\mathscr{F}_{s, t}$ for $|s| \leqslant \varepsilon,|t| \leqslant \varepsilon, \varepsilon>0$. The RHS of this formula denotes the endpoint of the geodesic segment starting at $f^{\alpha}(x)$ and determined by $s \mu^{\alpha}(x)+$ $t \nu^{\alpha}(x)$, where $\mu^{\alpha}=\mu \mid U_{\alpha}$ and $\nu^{\alpha}=\nu \mid U_{\alpha}$.

Received by the editors December 9, 1981.

1980 Mathematics Subject Classification. Primary 57R30.

Key words and phrases. Harmonic foliation, index.

'Work supported in part by NSF Grant MCS 79-00256. 
To explain the RHS in the second variation formula, observe that the canonical connection $\nabla$ in $Q$ defines an exterior differential $d_{\nabla}$ and codifferential $d_{\nabla}^{*}$ on $Q$-valued forms on $M$. Then $\Delta=d_{\nabla}^{*} d_{\nabla}$ is the Laplacian on the space of sections of $Q$, and the scalar product of sections $\mu, \nu \in \Gamma Q$ is given by

$$
\langle\mu, \nu\rangle=\int_{M} g_{Q}(\mu, \nu) \cdot \eta_{M}
$$

in terms of the canonical metric $g_{Q}$ on $Q$ and the volume form $\eta_{M}$ associated to the metric $g_{M}$ on $M$. The operator $\rho_{\nabla}: Q \rightarrow Q$ is the Ricci operator of the foliation, associated to the canonical metric and torsion free connection $\nabla$ in $Q$ (see [7] and §1 below).

This leads to the Jacobi operator of $\mathscr{F}[7]$ defined for $\mu \in \Gamma Q$ by

$$
g_{\nabla} \mu=\left(\Delta-\rho_{\nabla}\right) \mu \text {. }
$$

It is selfadjoint and strongly elliptic, thus with real eigenvalues

$$
\lambda_{1}<\lambda_{2}<\cdots<\lambda_{i}<\cdots \rightarrow+\infty \text { for } i \rightarrow \infty .
$$

The dimension of each eigenspace is finite, and the index of the foliation $\mathscr{F}$ occurring in the title is the sum of the dimensions of the eigenspaces corresponding to negative eigenvalues.

The purpose of this note is to prove the following result.

THEOREM. Let $\mathscr{F}$ be a Riemannian and harmonic foliation on the sphere $S^{n}(n>2)$ for which the standard metric is bundle-like. Then $\operatorname{index}(\mathscr{F}) \geqslant q+1$, where $q$ is the codimension of $\mathscr{F}$. Thus $\mathscr{F}$ is unstable.

The case $q=1$ does not occur for $S^{n}$, since a Riemannian foliation of codimension 1 is necessarily given by a closed nowhere zero 1-form [5, Proposition 3.14]. Thus $q \geqslant 2$ in the statement above. The case $q=n$ corresponds to the point foliation of $S^{n}$. Its index is the index of the identity map, which by Smith [9] equals $n+1$. This shows that the estimate above is best possible.

The positivity of the index was suggested by the corresponding result for harmonic maps on the sphere by Xin [10]. The two points to consider for the proof are the evaluation of the Ricci operator $\rho_{\nabla}$ of a harmonic foliation via the Weitzenböck formula ( $\$ 1)$, and the calculation of the Laplacian for sections of the normal bundle of $\mathscr{F}$ arising from the tangential projections of constant vector fields of $\mathbf{R}^{n+1}$ to $S^{n}$ (§2). We thank Luc Lemaire for the suggestion of the precise estimate $q+1$. In [3] the index of a harmonic map of maximal rank $q \geqslant 1$ on $S^{n}(n>2)$ is shown to be $\geqslant q+1$.

For the harmonic Hopf foliation $S^{1} \rightarrow S^{2 n+1} \rightarrow P^{n} \mathrm{C}$ the bound under discussion is $2 n+1$. It is of interest to note that Duchamp and Kalka [1] have shown that the space of holomorphic foliations near the Hopf foliation, i.e. foliations given by local submersions into $\mathbf{C}^{n}$ patching together via local biholomorphic transition functions $\mathbf{C}^{n} \rightarrow \mathbf{C}^{n}$, is already parametrized by a space of complex dimension $(n+1)^{2}$.

1. The Ricci operator of a harmonic foliation. Let $\mathcal{F}$ be a Riemannian foliation on $M$ given by $L \subset T M$. There is a unique metric and torsion free connection $\nabla$ in the normal bundle $Q=T M / L\left[5\right.$, Theorem 1.11]. Its curvature $R_{\nabla}$ is an $\operatorname{End}(Q)$-valued 
2-form on $M$. Since $i(X) R_{\nabla}=0$ for $X \in \Gamma L$, it follows that $R_{\nabla}(\mu, \nu): Q \rightarrow Q$ for $\mu, \nu \in \Gamma Q$ is a well-defined operator. Define $P_{\nabla}(\mu, \nu): Q \rightarrow Q$ by

$$
P_{\nabla}(\mu, \nu) s=-R_{\nabla}(\mu, s) \nu
$$

for $s \in \Gamma Q$. The Ricci curvature of $\mathscr{F}$ is then

$$
S_{\nabla}(\mu, \nu)=\text { Trace } P_{\nabla}(\mu, \nu)
$$

which is symmetric and bilinear. The Ricci operator $\rho_{\nabla}: Q \rightarrow Q$ is the corresponding selfadjoint operator given by

$$
g_{Q}\left(\rho_{\nabla} \mu, \nu\right)=S_{\nabla}(\mu, \nu)
$$

where $g_{Q}$ denotes the holonomy invariant metric in $Q$. In terms of an orthonormal basis $e_{p+1}, \ldots, e_{n}$ of $Q_{x}$ at some $x \in M^{n}$ we have

$$
\left(\rho_{\nabla} \mu\right)_{x}=\sum_{\alpha=p+1}^{n} R_{\nabla}\left(\mu, e_{\alpha}\right) e_{\alpha} .
$$

For more details see [7]. The underlying principle is that the usual curvature properties of a Riemannian manifold are well defined for a Riemannian foliation $\mathscr{F}$. They can be thought of as the corresponding curvature properties of a Riemannian manifold serving as model space for $\mathscr{F}$.

A metric $g_{M}$ together with the connection $\nabla$ gives rise to the usual calculus for $Q$-valued forms on $M$. In particular there are exterior differentials and codifferentials

$$
\begin{array}{ll}
d_{\nabla}: \Omega^{r}(M, Q) \rightarrow \Omega^{r+1}(M, Q), & r \geqslant 0, \\
d_{\nabla}^{*}: \Omega^{r}(M, Q) \rightarrow \Omega^{r-1}(M, Q), & r>0,
\end{array}
$$

and the Laplacian $\Delta=d_{\nabla} d_{\nabla}^{*}+d_{\nabla}^{*} d_{\nabla}$. This applies to the canonical projection $\pi$ : $T M \rightarrow Q$ viewed as $\pi \in \Omega^{1}(M, Q)$. In the following we assume $g_{M}$ to be bundle-like for $\mathcal{F}$, i.e. inducing $g_{Q}$ on $Q$. This defines a canonical metric splitting $\sigma: Q \rightarrow T M$ of $\pi$, so that $\sigma Q \cong L^{\perp}$. On $T M$ we consider the Riemannian connection $\nabla^{M}$ with curvature $R_{\nabla^{M}}$. At a point $x \in M$ we fix an orthonormal basis $e_{1}, \ldots, e_{n}$ of $T_{x} M$ with $e_{1}, \ldots, e_{p}$ in $L_{x}$ and $e_{\alpha}(\alpha=p+1, \ldots, n)$ in $(\sigma Q)_{x} \cong Q_{x}$. Let $E_{1}, \ldots, E_{n}$ be a local framing of $T M$ in a neighborhood of $x$ coinciding with $e_{1}, \ldots, e_{n}$ at $x$ and satisfying $\nabla_{e_{i}}^{M} E_{j}=0$. (We neither claim nor require that $\left(E_{i}\right)_{y} \in L_{y}$ for $1 \leqslant i \leqslant p$ or $\left(E_{\alpha}\right)_{y} \in \sigma Q_{y}$ for $p+1 \leqslant \alpha \leqslant n$ at $y \neq x$. But clearly $\left(\pi E_{i}\right)_{x}=\pi e_{i}=0$ for $1 \leqslant i \leqslant p$ and $\left(\pi E_{\alpha}\right)_{x}=e_{\alpha}$ for $p+1 \leqslant \alpha \leqslant n$.) The Weitzenböck formula evaluates the Laplacian $\Delta \pi \in \Omega^{\prime}(M, Q)$ as follows (see e.g. [8 or 6]):

$$
(\Delta \pi)_{x}=-\sum_{i=1}^{n} \nabla_{e_{i}} \nabla_{E_{i}} \pi+S(\pi)_{x}
$$

where for a vector field $X$

$$
S(\pi)_{x}(X)=\sum_{i=1}^{n}\left(R_{\nabla}\left(e_{i}, X\right) \pi\left(e_{i}\right)-\pi\left(R_{\nabla^{M}}\left(e_{i}, X\right) e_{i}\right)\right) .
$$

Only the value $X_{x} \in T_{x} M$ enters into this formula. With our choice of basis the first terms on the RHS disappear for $1 \leqslant i \leqslant p$. What remains is by (1.4) the term 
$\left(-\rho_{\nabla} \pi(X)\right)_{x}$. The second terms on the RHS add up to

$$
\pi\left(\sum_{i=1}^{n} R_{\nabla^{M}}\left(X, e_{i}\right) e_{i}\right)=\pi\left(\rho_{\nabla^{M}}(X)\right)_{x}
$$

where $\rho_{\nabla^{M}}$ denotes the Ricci operator of $g_{M}$, resp. $\nabla^{M}$. But for $M^{n}=S^{n}$ with its standard metric one has $\rho_{\nabla^{s}}=(n-1) \mathrm{Id}: T S^{n} \rightarrow T S^{n}$. Thus finally

$$
(\Delta \pi)_{x}=-\sum_{i=1}^{n} \nabla_{e_{i}} \nabla_{E_{i}} \pi-\left(\rho_{\nabla} \circ \pi\right)_{x}+(n-1) \pi_{x}
$$

as a map $T_{X} M \rightarrow Q_{x}$. The harmonicity of $\mathscr{F}$ is characterized by $\Delta \pi=0[5$, Theorem 3.3]. This yields the following result.

1.8 Proposition. Let $\mathcal{F}$ be a Riemannian and harmonic foliation on $S^{n}$ for which the standard metric is bundle-like. Let $\pi: T M \rightarrow Q$ be the canonical projection onto the normal bundle. Let $x \in S^{n}$ and $E_{1}, \ldots, E_{n}$ be a local frame of $T S^{n}$ on a neighborhood of $x$ such that $\left(E_{i}\right)_{x}=e_{i}$ form an orthonormal frame of $T_{x} S^{n}$ with $e_{1}, \ldots, e_{p} \in L_{x}$ and $e_{p+1}, \ldots, e_{n} \in \sigma Q_{x} \cong \sigma Q_{x} \cong Q_{x}$; and further $\nabla_{e_{i}}^{M} E_{j}=\left(\nabla_{E_{i}}^{M} E_{j}\right)_{x}=0$. For the Ricci operator of $\mathcal{F}$ we have then

$$
\left(\rho_{\nabla} \circ \pi\right)_{x}=-\sum_{i=1}^{n} \nabla_{e_{i}} \nabla_{E_{i}} \pi+(n-1) \pi_{x} .
$$

2. The Laplacian for special sections of $Q$. Let $X \in \Gamma T M$ and $\pi(X) \in \Gamma Q$. With the notations of the preceding section

$$
(\Delta \pi(X))_{x}=\left(d_{\nabla}^{*} d_{\nabla} \pi(X)\right)_{x}=-\sum_{i=1}^{n} \nabla_{e_{i}}\left(\nabla_{E_{i}} \pi(X)\right) .
$$

By the definition of the induced connections on $Q$-valued forms we have further

$$
\left(\nabla_{E_{j}} \pi\right)(X)=\nabla_{E_{j}} \pi(X)-\pi\left(\nabla_{E_{j}}^{M} X\right)
$$

so that

$$
(\Delta \pi(X))_{x}=-\sum_{i=1}^{n} \nabla_{e_{i}}\left(\left(\nabla_{E_{i}} \pi\right)(X)+\pi\left(\nabla_{E_{i}}^{M} X\right)\right) .
$$

Since

$$
\left(\nabla_{e_{i}}\left(\nabla_{E_{j}} \pi\right)\right)(x)=\nabla_{e_{i}}\left(\left(\nabla_{E_{j}} \pi\right)(X)\right)-\left(\nabla_{E_{j}} \pi\right)\left(\nabla_{e_{i}}^{M} X\right)
$$

we get

$$
\begin{aligned}
(\Delta \pi(X))_{x}= & -\sum_{i=1}^{n}\left(\nabla_{e_{i}} \nabla_{E_{i}} \pi\right)(X)-\sum_{i=1}^{n}\left(\nabla_{E_{i}} \pi\right)\left(\nabla_{e_{i}}^{M} X\right) \\
& -\sum_{i=1}^{n} \nabla_{e_{i}}\left(\pi\left(\nabla_{E_{i}}^{M} X\right)\right) .
\end{aligned}
$$

Note that the first sum occurs already in the formula (1.9) for the Ricci operator of $\mathscr{F}$. These terms therefore drop out for the Jacobi operator $\mathcal{G}_{\nabla}=\Delta-\rho_{\nabla}$.

The next step is to specialize (2.2) for the vector fields $V^{T} \in \Gamma T S^{n}$ arising from constant vector fields $V$ on $\mathbf{R}^{n+1}$ by tangential projection on $T S_{x}^{n}$ at each point 
$x \in S^{n}$. This means that for $x \in S^{n}$

$$
V_{x}^{T}=V-\langle V, N\rangle_{x} \cdot N_{x}
$$

where $N_{x}$ is the normal vector field defined by $N_{x}=x$.

2.4 Proposition. Let the situation be as in 1.8. Then for $X=V^{T} \in \Gamma T S^{n}$ we have

$$
\left(\Delta \pi\left(V^{T}\right)\right)_{x}=-\sum_{i=1}^{n}\left(\nabla_{e_{i}} \nabla_{E_{i}} \pi\right)\left(V^{T}\right)+\pi_{x}\left(V^{T}\right) .
$$

Proof. The connections $\nabla^{\mathbf{R}}$ in $\mathbf{R}^{n+1}$ and $\nabla^{S}$ in $S^{n}$ are related by the formula of Gauss

$$
\nabla_{e_{i}}^{\mathrm{R}} V^{T}=\nabla_{e_{i}}^{S} V^{T}+\alpha_{x}\left(e_{i}, V^{T}\right) .
$$

The second fundamental form $\alpha$ of $S^{n} \subset \mathbf{R}^{n+1}$ at $x$ is easily evaluated to be $\alpha=-g_{\mathbf{R}} \cdot N$, so that

$$
\alpha_{x}\left(e_{i}, V^{T}\right)=-\left\langle e_{i}, V^{T}\right\rangle \cdot N_{x}=-\left\langle e_{i}, V\right\rangle \cdot N_{x} .
$$

Using this and (2.3) one finds

$$
\begin{aligned}
\nabla_{e_{i}}^{S} V^{T} & =\nabla_{e_{i}}^{\mathbf{R}}(V-\langle V, N\rangle N)+\left\langle e_{i}, V\right\rangle \cdot N_{x} \\
& =-\langle V, N\rangle_{x} \cdot \nabla_{e_{i}}^{\mathbf{R}} N-e_{i}\langle V, N\rangle \cdot N_{x}+\left\langle e_{i}, V\right\rangle \cdot N_{x} .
\end{aligned}
$$

Note that for $\langle V, N\rangle_{x}=f(x)$,

$$
e_{i}\langle V, N\rangle=e_{i} f=d f_{x}\left(e_{i}\right)=\left\langle V, e_{i}\right\rangle \text {. }
$$

Further one verifies $\nabla_{X}^{\mathrm{R}} N=X$ for every $X \in \Gamma T S^{n}$, which expresses the fact that the Weingarten map of $S^{n} \subset \mathbf{R}^{n+1}$ equals -Id: $T S^{n} \rightarrow T S^{n}$. In particular $\nabla_{e_{i}}^{\mathbf{R}} N=e_{i}$ and thus

$$
\nabla_{e_{i}}^{S} V^{T}=-\langle V, N\rangle_{x} e_{i}
$$

Since $\nabla_{X}^{S} V^{T}$ depends linearly on $X \in T_{x} S^{n}$, it follows more generally that

$$
\nabla_{X}^{S} V^{T}=-\langle V, N\rangle X
$$

This formula implies further

$$
\left(\nabla_{E_{i}} \pi\right)\left(\nabla_{E_{i}}^{S} V^{T}\right)=-\langle V, N\rangle\left(\nabla_{E_{i}} \pi\right)\left(E_{i}\right) .
$$

But

$$
\left(\nabla_{E_{i}} \pi\right)\left(E_{i}\right)=\nabla_{E_{i}} \pi\left(E_{i}\right)-\pi\left(\nabla_{E_{i}}^{S} E_{i}\right) .
$$

For the framing chosen at $x$, the second term on the RHS vanishes at $x$. But this also holds for the value at $x$ of the first term. Namely if $1 \leqslant i \leqslant p$, then by the definition of the connection $\nabla$ in $Q[5,1.3]$

$$
\nabla_{e_{i}} \pi\left(E_{i}\right)=\pi\left[E_{i}, E_{i}\right]_{x}=0 .
$$

If $p+1 \leqslant \alpha \leqslant n$, then

$$
\nabla_{e_{\alpha}} \pi\left(E_{\alpha}\right)=\pi\left(\nabla_{e_{\alpha}}^{S} E_{\alpha}\right)_{x}=0
$$

Thus in any case

$$
\left(\nabla_{E_{i}} \pi\right)\left(\nabla_{e_{i}}^{S} V^{T}\right)=0
$$


Finally again by (2.7) and the results above

$$
\begin{aligned}
\nabla_{e_{i}}\left(\pi\left(\nabla_{E_{i}}^{S} V^{T}\right)\right) & =\nabla_{e_{i}}\left(-\langle V, N\rangle \pi\left(E_{i}\right)\right) \\
& =-\langle V, N\rangle_{x} \cdot \nabla_{e_{i}} \pi\left(E_{i}\right)-e_{i}\langle V, N\rangle \cdot \pi\left(E_{i}\right)_{x} \\
& =-\left\langle V, e_{i}\right\rangle \cdot \pi\left(E_{i}\right)_{x}=-\pi\left(\left\langle V, e_{i}\right\rangle e_{i}\right) .
\end{aligned}
$$

Since clearly $V_{x}^{T}=\sum_{i=1}^{m}\left\langle V, e_{i}\right\rangle e_{i}$ this shows that

$$
\sum_{i=1}^{n} \nabla_{e_{i}}\left(\pi\left(\nabla_{E_{i}}^{S} V^{T}\right)\right)=-\pi_{x}\left(V^{T}\right) .
$$

Substituting (2.8) and (2.9) in (2.2) yields (2.5).

3. The estimation of the index. Combining Propositions 1.8 and 2.4 we obtain for the Jacobi operator of a Riemannian and harmonic foliation $\mathscr{F}$ on $S^{n}$ the formula

$$
\mathcal{G}_{\nabla} \pi\left(V^{T}\right) \equiv\left(\Delta-\rho_{\nabla}\right) \pi\left(V^{T}\right)=(2-n) \pi\left(V^{T}\right) .
$$

For $n>2$ every nontrivial section of $Q$ of the form $\pi\left(V^{T}\right)$ for a constant vector field $V$ of $\mathbf{R}^{n+1}$ thus gives a 1-dimensional contribution to index( $\left.\mathscr{F}\right)$. It suffices to show that the space $\mho \subset \Gamma Q$ thus obtained satisfies

$$
\operatorname{dim} \psi \geqslant q+1 \text {. }
$$

Let $\mathscr{V} \subset \Gamma T S^{n}$ be the space of tangent vector fields of the form $V^{T}$ for $V \in \mathbf{R}^{n+1}$. Since $\mathbf{R}^{n+1} \rightarrow \mathcal{V}$ is clearly injective, $\operatorname{dim} \mathcal{V}=n+1$. Note that every vector field $V^{T}$ has exactly two zeros. Note further that the vector fields $V^{T}$ span $T_{x} S^{n}$ for any $x \in S^{n}$. Under the projection $\pi: T S^{n} \rightarrow Q$ clearly

$$
\begin{array}{lll}
\Gamma T S^{n} & \rightarrow & \Gamma Q \\
\cup & & \cup \\
\mathcal{V} & \rightarrow & \mho S \rightarrow 0
\end{array}
$$

Since the sections of $Q$ in $\mathscr{Q}$ necessarily span $Q_{x}$ for any $x \in S^{n}$, we have $\operatorname{dim}$ $\psi \geqslant q$.

It remains to show that the case $\operatorname{dim} \mho \widetilde{W}=q$ is not possible. Namely assuming this, there is a basis $X_{1}, \ldots, X_{n+1}$ of $\mathcal{V}$ such that the vector fields $X_{1}, \ldots, X_{n+1-q}$ span the kernel of $\mathcal{V} \rightarrow \mathscr{W}$ and the remaining $q$ vector fields map onto a basis $Y_{1}, \ldots, Y_{q}$ of $W$. Since the $Y_{1}, \ldots, Y_{q}$ generate $Q_{x}$ in every point $x \in S^{n}$ and $q=\operatorname{dim} Q_{x}$, none of the $Y_{j}$ can ever vanish. But the corresponding $X$ 's in $\mathcal{V}$ are then a fortiori nonsingular vector fields on $S^{n}$. Since by construction all elements of $\mathcal{V}$ do have zeros, this contradiction eliminates the case $\operatorname{dim} \mho=q$. Thus $\operatorname{dim} \mho \geqslant q+1$ which finishes the proof.

\section{REFERENCES}

1. T. Duchamp and M. Kalka, Holomorphic foliations and deformations of the Hopf foliations, preprint.

2. J. Eells and J. H. Sampson, Harmonic mappings of Riemannian manifolds, Amer. J. Math. 86 (1964), 109-160.

3. J. Eells and L. Lemaire, Selected topics in harmonic maps, CBMS Regional Conf. Ser. in Math., Amer. Math. Soc., Providence, R. I. (to appear).

4. F. W. Kamber and Ph. Tondeur, Feuilletages harmoniques, C. R. Acad. Sci. Paris Ser. A-B 291 (1980), 409-411. 
5. 1980.

6. Curvature properties of harmonic foliations (to appear).

7. Infinitesimal automorphisms and second variation of the energy for harmonic foliations, Tôhoku Math. J. 34 (1982).

8. Y. Matsushima, Vector bundle valued harmonic forms and immersions of Riemannian manifolds, Osaka J. Math. 8 (1971), 1-13.

9. R. T. Smith, The second variation formula for harmonic mappings, Proc. Amer. Math. Soc. 47 (1975), 228-236.

10. Y. L. Xin, Some results on stable harmonic mappings, Duke Math. J. 47 (1980), 609-613.

DeParTMENT OF MATHEMatics, UNIVERSITY OF ILLINOIS, URbaNa, ILLINOIS 61801 